\title{
Estudiando en igualdad de condiciones: curso universitario para estudiantes de trabajo social y usuarios de servicios ${ }^{1}$
}

\author{
Verner Denvall, Cecilia Heule, Arne Kristiansen*
}

\begin{abstract}
Resumen
Es crucial para la formación en Trabajo Social proveer las oportunidades para que los estudiantes alcancen un conocimiento sincero y genuino de las condiciones de la vida que son comunes para las personas con las que trabajarán. Los estudiantes de Trabajo Social y los usuarios de servicios sociales con experiencias personales y problemas sociales múltiples ahora se reúnen como participantes en un curso experimental en la Escuela de Trabajo Social en Lund, Suecia. En este artículo, los profesores entregan sus experiencias.
\end{abstract}

\section{Palabras clave}

Formación en Trabajo Social, Estudiantes de Trabajo Social, Usuarios de Servicios, Problemas Sociales, lgualdad de Oportunidades.

\section{Abstract}

It is crucial for social work education to provide opportunities for students to gain a sincere and genuine knowledge of the conditions of life that are common for their forthcoming clients and patients. Social work students and service users with experiences of multiple social and personal problems now meet, as they are participants in an experimental course at the School of Social Work in Lund, Sweden. In this paper the teachers report their experiences.

\section{Key words}

Social Work Education, Social Work Students, Services Users, Social Problems, Equal Terms

\section{Presentación}

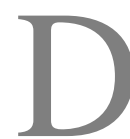
esde el otoño de 2005, la Escuela de Trabajo Social de la Universidad de Lund, Suecia, ha dictado un curso titulado "Movilidad Social, Trabajo Comunitario y Cambio Estructural”. El curso se da por seis semanas y forma parte del último semestre de la formación de un trabajador social ${ }^{2}$. Uno de los objetivos del curso es que los estudiantes aprendan sobre formas sostenibles de integración y de cambio social. El curso está dirigido a estudiantes -de trabajo social- que están cursando la carrera, así como para personas que se reclutan de varias organizaciones de servicio público.

2 El Trabajo Social universitario que se enmarca en las Ciencias Sociales, se estudia en 3.5 años, debiendo aprobarse 140 créditos, 1 crédito sueco es igual que 1.5 créditos de ECTs. En el año 2006, el Trabajo Social se puede estudiar en 21 universidades y universidades-colleges en Suecia. 
El curso es opcional y elegido por los estudiantes como módulo en el semestre final.

$\mathrm{Al}$ reclutar a personas usuarias de servicios públicos, una diversidad de grupos que arriesgan ser marginados en la sociedad Sueca se esfuerzan por quedar. Ejemplos de estos estudiantes son personas pertenecientes a minorías étnicas, otras con antecedentes de adicción a las drogas, así como también gente con varias formas de discapacidad y desórdenes psiquiátricos. Estas personas una vez integradas al programa, son denominadas por nosotros, como estudiantes usuarios de servicio público. Podemos admitir a cerca de 15 estudiantes usuarios de servicio público y a 30 estudiantes de Trabajo Social cada semestre.

Este tipo de curso, por lo que sabemos, es único, en el sentido que se dirige a grupos a los que generalmente no se les da la oportunidad de reunirse en igualdad de condiciones en un contexto educativo común. Además, la participación de los usuarios de los servicios dentro de la educación de trabajadores sociales, está hasta ahora relativamente no probada y se da sólo esporádicamente dentro de la educación de Trabajo Social Sueca (Socialstyrelsen 2003).

Aunque se contrata a los usuarios del servicio público como conferencistas, ésta es una forma pasiva de participación. Incluso si la incorporación del usuario del servicio público es algo más común en la educación del Trabajo Social, como lo es en algunos países, por ejemplo Gran Bretaña, donde ésta consiste casi exclusivamente en el uso de diversos representantes del servicio como conferencistas (véase por ejemplo Levin 2004, publicado en el Instituto Social del Cuidado para la Excelencia, SCIE).

Durante las primeras dos semanas del curso, a todos los estudiantes se les da la ocasión de compartir sus experiencias de vida, a través presentaciones orales. Además se dictan conferencias sobre el autocontrol del destino de las comunidades, movilidad social y empresa social. Después de esto, el programa se centra en un curso denominado "Taller Futuro", que corresponde a un método para desplegar y realizar ideas, donde el empeño y la creatividad se combinan con un espíritu de trabajo democrático y enérgico, donde el encanto y la creatividad se combinan con el trabajo conducido por la acción (Jungk y Müllert 1984, Denvall y Salonen 2000). En este curso, los estudiantes forman grupos de trabajo según sus intereses. Cada grupo propondrá planes innovadores para el desarrollo del Trabajo Social práctico, en dos semanas. Los grupos de trabajo presentan sus proyectos ante una comisión que consiste en políticos influyentes e investigadores, que evaluarán críticamente la importancia y la viabilidad de los proyectos. Durante el tiempo que los estudiantes trabajan con sus proyectos, ellos también asisten a conferencias acerca del fenómeno que están abordando.

Además de otorgar a los estudiantes la posibilidad de alcanzar un conocimiento profundo sobre tales temas, nosotros, como docentes, vemos esta experiencia como un desafío y una posibilidad para la creación de nuevos lugares de encuentro entre usuarios de servicios y estudiantes, para realizar investigaciones y prácticas.

Somos críticos de las construcciones sociales que sustentan los problemas sociales y sus soluciones, con las que se tiende a formar a los estudiantes del Trabajo Social. A través del contacto cercano que el curso ofrece entre las personas que han experimentado la discriminación y la exclusión y los futuros profesionales con compromiso social, emergen encuentros inesperados, así como también nuevas posibilidades.

El curso es dictado por cinco semestres y se realiza en forma de proyecto, financiado por los fondos estructurales de la Unión Europea a través del Programa de Igualdad. ${ }^{3}$ Siendo evaluado continuamente, en parte por los profesores del curso, y sometiéndose a escrutinio independiente. El trabajo

3 Para más detalles ver: http://www.esf.se/esf/templates/ Page. aspx? id $=607$ 
de evaluación reúne, entre otras cosas, el background o la experiencia previa de los estudiantes, sus expectativas frente al curso, así como lo que han aprendido y su experiencia en general.

El propósito de este artículo es describir y poner en discusión algo de lo que hemos experimentado durante los primeros dos semestres del curso. Lo que hasta ahora ha sido una experiencia muy positiva. La forma es innovadora y favorece no solamente a estudiantes de Trabajo Social, sino también a estudiantes usuarios de organizaciones de servicio, junto con tales organismos. A través de varias aproximaciones pedagógicas los participantes del curso emergen como individuos complejos, y esto contribuye a una comprensión más profunda y a una interpretación más personal, que las categorías rígidas anteriores: estudiante de trabajo social, usuario de un servicio, ex adicto, muchacha joven, gitanos y musulmanes, por ejemplo.

Que nuestras experiencias hayan sido positivas no implica que el curso esté libre de desafíos y de problemas. Incluso habiéndonos preparado para ello, en ocasiones hemos sido sorprendidos por las sensaciones que el curso ha provocado en algunos de los estudiantes.

\section{El curso como proyecto en un discurso circundante}

Hay un aumento del interés en la utilización del conocimiento y de las historias de la gente vulnerable, en la investigación y el desarrollo del conocimiento en el Trabajo Social (Beresford et al., 1999; Krumer-Nevo, 2005). Michael Krumer-Nevo (2005) en su artículo "Escuchando al conocimiento de la vida: una nueva dirección de la investigación en estudios de pobreza” da ejemplos de Israel, Bélgica y de los E.E.U.U. de cómo los investigadores trabajan en conjunto con las experiencias de vulnerabilidad propias de la gente. Una cita de Tardieu abre su artículo:

“Descubrimos que lo principal no era dar a los pobres la oportunidad de hacer escuchar su voz, sino que abrir nuestros oídos. No se trata de darle poder a los pobres, sino de humanizar a ciudadanos e instituciones.” (Tardieu 1999:75)

Esta cita se puede utilizar no solamente como punto de partida para una discusión crítica sobre cómo se organiza y se lleva a la práctica el Trabajo Social, sino que también cómo se comunica en la educación del Trabajo Social. A pesar del sinnúmero de indicadores de que la marginalización y las causas estructurales, han conducido cada vez más a los problemas sociales durante las últimas décadas, muchos investigadores han determinado que ha habido una individualización tanto de la asistencia social, como de la búsqueda de soluciones para varios problemas sociales (Järvinen y Mik-MikMeyer, 2003). Además, la profesionalización del Trabajo Social ha implicado la burocracia, que tiende a separar al profesional de la perspectiva del usuario. Salonen (1998) dice que el ser cliente de un servicio implica estar en un estado de dependencia de una organización por sobre la que el individuo no tiene influencia alguna. Kristiansen $(1999,2005)$ describe cómo los practicantes se caracterizan por tener un punto de vista orientado a las carencias y a los problemas de los usuarios de servicios.

Esto crea clasificaciones y una construcción de roles estereotipados que a menudo mantienen una idea de nosotros-y-ellos conectada con las construcciones sociales. Sostenemos que esto también se refleja en la educación del Trabajo Social en Suecia. La falta de participación en la educación del Trabajo Social (Socialstyrelsen, 2003), a la que nos referimos en la introducción, se puede considerar como expresión de esto.

Pensamos que los cursos de "Movilidad Social, Trabajo Comunitario y Cambio Estructural” implican una interpretación crítica del Trabajo Social. A través del trabajo en conjunto entre los estudiantes usuarios de servicios y de los estudiantes de Trabajo Social, la perspectiva del usuario del servicio, se trata en la educación de manera totalmente diferente e integrada, en comparación a cuando es

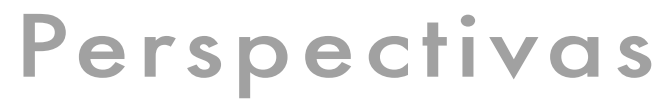


comunicada solamente con charlas separadas dadas por los usuarios de servicio invitados. Este cambio en el discurso se puede comparar con la descripción de Adán (2003) de cambio de paradigma en Trabajo Social, donde el uso de poder se describe como una nueva perspectiva que sustituye un viejo paradigma, en el cual antiguamente, el trato a los clientes era lo esencial en el Trabajo Social.

Existen semejanzas entre el diseño del curso y la descripción hecha por Gutiérrez (1990) que contempla cinco pasos en el proceso hacia el uso del poder: aceptar la definición de un problema desde el punto de vista del cliente; identificar y construir sobre las fortalezas; trabajar en el análisis de la situación del cliente desde una perspectiva del poder; enseñar habilidades específicas como también los recursos de movilidad para el cliente y cautelarlos.

Jan Fook (2002) describe cómo las diferentes perspectivas, tales como, la del usuario de servicio y la perspectiva profesional se arraigan en distintas lógicas. Asimismo, el enfoque científico y el de un practicante, representan diversos discursos que co-existen temporalmente, pero rara vez se encuentran. Además, a partir de los objetivos que apuntan al cliente, antes mencionados (Gutiérrez, 1990), el curso intenta crear una plataforma para estimular el diálogo entre la gente cuyos discursos son de un carácter diverso.

La práctica demuestra que los efectos sinérgicos que se suceden actúan para movilizar no sólo a aquellos con la experiencia de discriminación, sino también a los estudiantes que no comparten esas experiencias.

\section{La paradoja de la categorización}

Existe una paradoja entre el diseño y las metas del curso, ya que, durante la fase de incorporación de los estudiantes usuarios de servicios, usamos lo que se puede describir como maneras rígidas $\mathrm{y}$ unilaterales de categorizar a la gente. A fin de ser elegible para el curso, una persona puede ser un gitano, un inmigrante, o puede tener antecedentes de alguna adicción o secuelas de una enfermedad psiquiátrica o física. El hecho que nosotros captemos alumnado sobre la base de estos tipos de clasificación es debido a que nos vemos enfrentados a una multiplicidad de perspectivas de discriminación y de lo distinto; por lo tanto, este punto se convierte en un objetivo importante para flexibilizar estas clasificaciones rígidas.

Implica un desafío para nosotros como profesores, contribuir a que los estudiantes se aventuren fuera de sus clasificaciones, formen nuevas agrupaciones y encuentren camaradería más allá de sus categorizaciones originales. Un ejemplo de cómo el curso desafía estas clasificaciones y desarrolla afinidad y solidaridad quedó en evidencia cuando una mujer con una discapacidad física recitó sus poemas que versaban sobre el ser extranjero, y muchos estudiantes expresaron lo emocionados que se habían sentido. Un gitano preguntó si uno de los poemas se trataba sobre los gitanos, y con esto él manifestaba que la escritora tenía una visión de gran alcance respecto de su situación y experiencias.

A través de esta metodología, los estudiantes de Trabajo Social, así como aquellos usuarios de servicios, son capaces de trabajar juntos y compartir historias personales. A partir de ello, surge una sensación de afinidad con toda la humanidad en lo macro. Lo "cotidiano" es discutido por los estudiantes usuarios de servicios, junto con las experiencias de vulnerabilidad que emergen desde lo conocido por los estudiantes de Trabajo Social. En una reflexión escrita un estudiante resumió la paradoja del curso de la siguiente manera:

"Algo que veo como importante a considerar es que el individuo puede sólo hasta cierto punto representar y ser el portavoz de su grupo. La experiencia de diversos acontecimientos es subjetiva y puede variar enormemente incluso dentro de lo que puede parecer ser un grupo homogéneo. Por ejemplo, puede que una persona lisiada nunca diga que sabe cómo otras personas lisiadas sufren su 
situación y así sucesivamente. Ha sido de gran interés seguir los diferentes puntos de vista que se encuentran dentro de los varios grupos representados. Con esto quiero decir que es importante tener una actitud humilde cuando uno se enfrenta a diversas interpretaciones de situaciones, y ello es algo que este curso me ha enseñado. Creo que he visto que los individuos en lo que parece ser un grupo homogéneo, son menos semejantes que lo que uno creería, al mismo tiempo, nosotros como personas somos más parecidos que lo que generalmente creemos. Es interesante ver lo semejante que somos, a pesar de todas las diferencias...”

A fin de clarificar esta paradoja de la clasificación de manera teórica, el concepto inter-seccional (De Los Reyes y Mulinari, 2002; De Los Reyes, 2005) es interesante, además de aplicable. Al usarlo como un punto de partida, uno puede decir que la comprensión de la vulnerabilidad exige el conocimiento que va más allá de clasificaciones unidimensionales y sobre-simplificadas.

\section{Ideas y aprendizajes}

Los estudiantes son semejantes en el curso, sin embargo, cuando reflexionan acerca de la evaluación del curso, hay algunas diferencias entre los estudiantes de Trabajo Social y quienes son usuarios de servicios.

Muchos de los alumnos de usuarios del servicios, destacan el aumento de la confianza en si mismos, lo que puede estar conectado con la realización de un curso universitario. Ellos subrayan la importancia de ser capaces de relacionar sus experiencias, con la de los futuros trabajadores sociales, además de desarrollar nuevas relaciones y una comprensión en profundidad de lo que involucra el sentarse al otro lado del escritorio en el rol de "ayudante". $\mathrm{Al}$ mismo tiempo, cabe destacar la magnitud del desafío que esto representa para el grupo, en el que muchos llevan consigo la experiencia de fracasos anteriores en la escuela o en el trabajo, algo que no debe ser subestimado. La mayoría, por ejemplo, no ha sido consciente de cuanto tiempo les tomará estudiar, de que deben pasar mucho tiempo en la universidad y además de la autodisciplina que se requiere para comprender la literatura obligatoria y la elaboración de artículos para los exámenes ${ }^{4}$. Hemos, por lo tanto, reservado recursos especiales en forma de mentores, de manera que los alumnos tengan la posibilidad de recibir apoyo y tuición extra. $^{5}$

Por su parte, los estudiantes de Trabajo Social destacan la importancia de los encuentros con personas cuyas experiencias son a menudo dolorosas, tales como ser discriminados por las autoridades. Además resaltan que la construcción de la noción de "cliente", a menudo rígida, que hasta ese entonces ha sido llevada por la educación, es desafiada. Para muchos estudiantes de Trabajo Social, el curso incluye una re-evaluación de esa imagen, la que fue creada durante los semestres anteriores, así como de su propia identidad dentro de la disciplina.

El curso, a menudo, por lo menos al inicio, incluye para muchos un proceso donde los prejuicios y las ideas pre-concebidas son desafiadas, y esto induce un proceso de reflexión sobre cómo uno se conduce en la práctica del Trabajo Social.

4 Los requisitos para aprobar el curso pueden ser resumidos del siguiente modo: Tiempo completo de estudios, lo que implica estudio individual luego de las horas en la universidad, lo que incluye 900 páginas de literatura Sueca e Inglesa, asistencia obligatoria de tres a cinco días a la semana, un informe de examen final de 4 páginas, una presentación de dos horas de la organización a la que representan, del proyecto del grupo y la presentación del plan de proyecto a una comisión evaluadora.

5 Además de la tuición que se ofrece para los estudiantes de trabajo social, la que incluye, entre otras cosas, talleres y acceso libre a diccionarios, salas de computación con Internet y bibliotecas, los usuarios del servicio también tienen acceso a: entrenamiento individual o grupal para el estudio de la literatura, entrenamiento individual para la elaboración del informe examen, apoyo de conversación para potenciar la reflexión durante el curso, supervisión grupal, guía en la preparación de la presentación de la organización a la que representan, resúmenes en Sueco de la literatura en Inglés junto con una lista de palabras en Sueco y en Inglés sacadas de la literatura del curso. 
Un alumno de Trabajo Social lo describió de la siguiente manera:

"Sin entender muy bien por qué era, yo frecuentemente me sentía provocado y bastante irritado cuando viajaba a casa desde la universidad, espacialmente al inicio del curso. A veces tenía el sentimiento de que muchos de los usuarios del servicio pensaban que tenían conocimiento exclusivo de cuáles eran las necesidades de la gente socialmente vulnerable y que las soluciones definitivamente no venían desde la Escuela de Trabajo Social... El curso primeramente me hizo mirarme y conectar las cosas conmigo mismo. ¿Por qué me provocaban? La universidad es un contexto del que tengo ciertas expectativas y uno se comporta de cierta manera. Me sentía como si mucho de mi conocimiento se transformara en realidad en compañía de los estudiantes usuarios de servicios públicos y he aprendido cosas que no aprendí en cátedras normales.”

El curso obliga a los estudiantes a dar un paso fuera de su “zona de confort” y, por lo tanto, a tener que gastar mucha energía para comprender y demostrar deferencias con "el otro".

Por otra parte, para los docentes también ha sido una experiencia enriquecedora:

"Siento que el curso ha sido difícil de una manera distinta a otros cursos anteriores, porque ha requerido algo más de mi. Por mi lado, siento que he dedicado bastante tiempo en tratar de entrenar a los estudiantes y en asegurarme de que ellos sean parte del grupo, tanto en la sala de clases como en los grupos pequeños. Eso ha significado que he invertido más de lo que se había requerido anteriormente en mi práctica docente, y esto ha hecho que me sienta con sobre carga de trabajo. Sin embargo, pienso que ha sido muy entretenido y que he aprendido mucho. Tanto acerca de mi misma, como de toda la gente interesante que han osado aventurarse en este cerrado mundo de la educación superior, tan valientes.”

\section{El objetivo de la diversidad no es el consenso}

Existe una tendencia a las diferencias de opinión, que uno no puede, o no debe, esforzarse en suprimir. En lugar de eso, es pertinente clarificar los tipos de oposición que pueden presentarse cuando se reúne a personas con diversas normas acerca de lo que se puede decir en una reunión pública. Durante el primer curso, que se llevó a cabo en el otoño de 2005, los estudiantes de Trabajo Social nos contactaron, en numerosas ocasiones, a nosotros los profesores, para mostrar su descontento porque los estudiantes usuarios de servicios no tenían la opinión "correcta”. Uno de ellos indicó que sentía que sus derechos estaban siendo vulnerados, esto es interesante, pues cuando la vulneración a los derechos se discute dentro del Trabajo Social, son generalmente los derechos de los usuarios de servicios los que son considerados como infringidos.

Durante el segundo curso (primavera del 2006) hemos tenido mayor éxito en crear una atmósfera permisiva y un nivel más alto de tolerancia. Una estudiante de Trabajo Social describió un encuentro significativo durante el curso de la siguiente manera:

"Camino a mi casa en Malmö en el tren, le pregunté a Moussad acerca de su esposa. Sabía que se habían casado hace bastante tiempo en un país del Medio Este, pero que ella todavía no había venido a Suecia, y esto me causaba curiosidad. ¿Cómo se habían conocido? ¿Era un matrimonio acordado? ¿Cómo se sentía saber que pronto se reunirían? Moussad me dijo que le presentaron a su esposa, quien pertenece a la misma tribu que él, cuando él visitó sus parientes en su patria, se dieron cuenta de que se gustaron y después de haberse conocido por cinco meses se casaron. Era una unión acordada pero con amor, pensé con alivio. Esto fue hace dos años, y ahora, por fin, le habían dado un permiso de residencia en Suecia. Moussad parecía contento y ansioso cuando explicó que su esposa 
estaría llegando en algunas semanas. Después de esto, él me preguntó que si tenía un novio. Tengo una novia le contesté. Moussad me miró con una sonrisa y no sabía qué decir. Vi por su expresión que esto era algo muy grande para él. Él probablemente nunca había conocido a alguien homosexual antes. Moussad pronto pensó en algo que decir, pero dijo que no estaba seguro de expresarlo por miedo a ofenderme. Te respeto por lo que eres, dijo él varias veces. Esta reunión ilustra para mí las seis semanas que este curso abarcó. He conocido a gente que probablemente nunca habría tenido la ocasión de conocer. Había varios puntos de partida, pero los encuentros que se crearon por semanas, formaron relaciones en las que las personas con las que estuve, y yo, estábamos al mismo nivel”.

Las reuniones que se realizaron en la sala de clases abrieron espacios para pensar que no solo había diferencias entre grupos de estudiantes usuarios de servicios y estudiantes de Trabajo Social, sino que somos todos diferentes como individuos, y esta idea, por otro lado, conduce a que, de hecho, por ser todos diferentes, somos todos iguales.

"Estos encuentros han implicado una prueba a mis prejuicios, valores y conocimientos. Los días han sido largos e intensos, por las tardes estoy cansado. La reflexión intensa y constante ha caracterizado este período. Naturalmente, he aprendido mucho de esto, tanto para mi como persona, como a nivel profesional. Un proceso se ha puesto en movimiento, un proceso de reflexión, que creo, puede continuar en el futuro, y especialmente durante el tiempo en que comience a desarrollarme como Trabajador Social”.

Durante el segundo curso tanto los estudiantes usuarios de servicio, como los estudiantes de Trabajo Social, han sido extremadamente generosos en compartir historias sobre sí mismos que han apuntado a diversas direcciones. Hay un consenso acerca de, por ejemplo, la importancia de ser tratado amablemente, participación y motivación, pero, por sobre todo, hubo una gran diversidad entre las visiones sostenidas por los estudiantes. Lo anterior demuestra cómo la gente con diversos puntos de vista y perspectivas distintas han podido cooperar en diversos proyectos a pesar de las disimilitudes y contrastes de opinión.

\section{El arte de la movilidad sin infringir los derechos de otros}

Para dirigir un curso que intenta ser significativo para personas de diferentes culturas, de organizaciones que representan diferentes -y a veces opuestas- perspectivas, se requiere liderazgo. El objetivo de tal liderazgo es capturar las tensiones y ser un estabilizador en un océano de experiencias personales. De ese modo, el curso se convierte en un ejemplo de las dificultades con que el trabajo de movilización social se enfrenta a menudo.

Las personas quieren tener un rol, es decir, la necesidad de auto realizarse es innata y tangible para un estudiante que casi ha completado su educación, y pronto comenzará su vida como trabajador. Durante el primer curso, aprendimos cuán importante era, tanto para estudiantes de Trabajo Social como para estudiantes de servicios, tener la oportunidad de desarrollar un positivo e importante rol durante su curso. Que esto demandara tanta planificación fue algo que habíamos subestimado. Los Trabajadores Sociales tienden a reflexionar muy poco sobre qué tan importante es para el cliente alcanzar otro rol más productivo que el de ser cliente.

Permitir a todos dar un paso hacia adelante como individuos contrarrestó la tendencia que se dio durante el segundo curso, de disputas entre la perspectiva del profesional y la perspectiva del usuario de servicios. Para poder contrarrestar el estereotipo de "nosotros y ellos" y para tener éxito en la integración, se necesitaba que los actores se convirtieran en algo más que los estereotipos comunes.

La experiencia dice que muchos de los estudiantes usuarios de servicios ven el curso como un gran reto, en parte por dificultades previas que los han

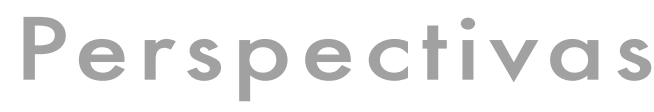


llevado a no atreverse a elevar sus expectativas. Una joven discapacitada mental describió la manera en que se sentía antes de embarcarse en el proyecto, de la siguiente manera:

"La ventaja con el curso es que los estudiantes de Trabajo Social y los estudiantes usuarios de servicios, intercambian y comparten sus trabajos y situaciones de la vida cotidiana. Esto se aclaró durante el proyecto de trabajo. Teníamos como meta dar empleo a las personas con un historial extranjero a través del uso de una empresa social. Mis compañeros dijeron: "Por supuesto que las personas que han estado desempleadas por un largo tiempo, son felices cuando una empresa social se atreve a invertir”. Mi experiencia y el miedo a la desilusión, hicieron que soñar aquello fuera doloroso. También sé que las personas quizás necesiten apoyo terapéutico y que toma tiempo sanar como persona. Si hubiésemos tenido más tiempo podríamos haber reflexionado sobre nuestras propias experiencias después. Fue bueno para mí tener un horario que seguir, sin tener que diseñarlo yo misma. A lo largo del curso mis experiencias difíciles se habían alejado"

Sin embargo, lo que nos ha llamado la atención cuando hemos observado las necesidades de los estudiantes usuarios servicios, ha sido que los estudiantes de Trabajo Social, habían pedido apoyo para sí y para ellos, debido a que el curso cubre un terreno que ellos no esperaban.

Hemos visto la importancia de la búsqueda creativa de formas pedagógicas de reforzamiento de la autoestima de los estudiantes de Trabajo Social y de su apego a la disciplina. Esto se potencia a través del contacto que se establece entre los estudiantes y con aquellos que han sido desilusionados e insultados por las autoridades locales.

El curso en cuestión también ha reportado experiencias de aprendizajes para los profesores. Uno de nosotros lo ha resumido de la siguiente manera:

"Me he enfrentado al reto no menor de crear con- fianza, en poco tiempo, en personas cuyas experiencias han sido testigos del problema de confiar en otros y en sí mismos, con las dificultades que ello implica. El desafío personal es comprender los esfuerzos de las personas, que básicamente se centra en lograr ser vistos y tratados de la misma manera que todos los demás. Ello implica no solamente centrarnos en el enojo hacia las organizaciones discriminatorias de la sociedad, sino que además, esta experiencia despierta un gran deseo y voluntad de cambio, no solamente para estos individuos específicos, sino que también para los grupos que ellos representan. Durante el curso, he compartido tragedias humanas que testifican los de afuera, y además he llegado a conocer a estas personas que, contra todo pronóstico, han reclamado poder sobre sus vidas, han obtenido victorias, y se han fortalecido gracias a la adversidad. Ha sido muy enriquecedor ser capaz de compartir en este trabajo práctico y ver muy de cerca cómo las personas crecen y se desarrollan.” (Ottengrim 2006).

\section{Reflexión final:}

Percibimos que este tipo de curso es un ejemplo innovador que, de una manera radical, muestra las nuevas maneras de cambiar perspectivas y permitir a los estudiantes confrontar sus propios prejuicios y retar sus habilidades. Este curso puede, también, proveer miradas introspectivas e inesperadas y animar a un grupo de personas, a los estudiantes usuarios de servios, que hasta ahora han sido removidas de la educación superior, a integrarse a ella. Hemos aprendido mucho de los dos cursos que han sido realizados y es urgente que estas experiencias sean un aporte en otras escuelas en Suecia. Por lo tanto, queremos estimular más iniciativas e incluir la participación de usuarios de servicios en la agenda pedagógica de una manera tangible. De este modo, podemos contribuir en la formación de Trabajadores Sociales más juiciosos y atentos.

En un intento por difundir el conocimiento, hemos ofrecido a otras escuelas de Trabajo Social de Suecia, un foro de discusión con actores locales, 
como profesores, estudiantes y organizaciones de servicios. Participar en el congreso mundial de Trabajo Social es otra oportunidad para permitir que el proyecto sea expuesto a trabajadores sociales, investigadores y docentes vinculados a la profesión. Por supuesto, deben existir vías alternativas para fomentar la participación de usuarios de servicios en la educación de Trabajo Social, lo que debería ser puesto en discusión.

Una cosa que puede ser asegurada antes de comenzar cada curso es que lo impredecible que éste será cuando se trata de estudiantes muy diversos en experiencias en la vida. Al comienzo de cada curso, aparecerán situaciones con las que se deberá

\section{BIBLIOGRAFÍA}

ADAMS, R. (2003) Social Work \& Empowerment. New York: Palgrave.

BERESFORD, P; GREEN, D; LISTER, R y WOODARD, K. (1999) Poverty First Hand: Poor people speak for themselves. London: CPAG.

DE LOS REYES, P y Kamali, M. (red) (2005) Bortom vi och dom. Teoretiska reflektioner om makt, integration och strukturell diskriminering. SOU 2005:41.

DE LOS REYES, P y MULINARI, D. (red) (2002) Maktens (o)lika förklädnader. Kön, klass och etnicitet i det postkoloniala Sverige. Stockholm: Atlas.

Denvall, V y Salonen, T. (2000) Att bryta vanans makt. Framtidsverkstäder och det nya Sverige. Lund: Studentlitteratur.

FOOK, J. (2002) Social Work: Critical Theory and Practice. London: Sage.

GUTIÉRREZ, L. M. (1990) Working with Women of Color: An Empowerment Perspektive. Social Work 35(2): 149-153.

JUNGK, R y MÜLLERT, N (1984) Håndbog i fremtidsvcerksteder. København:Politisk Revy.

JÄRVINEN, M y MIK-MEYER, N (red)(2003) At skabe en klient: institutionelle identiteter $i$ socialt arbejde. Köpenhamn: Hans Reitzel. lidiar. Sin embargo, si tales eventos son manejados de buena manera, pueden ofrecer una posibilidad para reflexionar y aprender lo que no se puede aprender a través de lecturas. Los cursos de este tipo representan, como los mismos participantes, a la sociedad en general.

Esta diversidad puede ser vista como un motor y fuente de inspiración para las nuevas maneras de pensar. Más aún si consideramos que durante el curso se establecen puntos de contacto que abarcan a un gran número de organizaciones, lo que implica que la creación de nuevos proyectos e idas. Ha sido, por tanto, una plataforma dinámica e inspiradora, tanto para profesores como para estudiantes, ya que los cursos se han desarrollado de modos que nunca hubiésemos podido predecir al comienzo.

KRISTIANSEN, A (1999) Fri från narkotika. Om kvinnor och män som har varit narkotikamissbrukare. Umeå: Studier i socialt arbete $\mathrm{nr} 28$, Institutionen för socialt arbete, Umeå universitet.

KRISTIANSEN, A. (2005) Flickor i tvångsvård. Utvärdering av tolvstegsinriktad §12-vård. Stockholm: Statens institutionsstyrelse.

KRUMER-NEVO, M (2005) "Listening to life knowledge: a new research direction in poverty studies". International Journal of Social Welfare. Volume 14, nr 2.

LEVIN, E (2004) Involving Service Users and Carers in Social Work Education. London: SCIE 2004:2.

OTTENGRIM, A (2006) Reflektioner kring kursen Social mobilisering och förändringsarbete utifrån ett mentorsperspektiv. Lund: Socialhögskolan.

SALONEN, T. (1998) KLIENT, DENVALL, V. Y JACOBSON, $\mathrm{T}$ (red), Vardagsbegrepp i socialt arbete. Ideologi, teori och praktik. Stockholm: Norstedts Juridik.

SOCIALSTYRELSEN (2003) Brukarmedverkan i socialtjänstens kunskapsutveckling. Stockholm: Socialstyrelsen. The National Board of Health and Welfare, (www.sos.se).

TARDIEU, B. (1999) "Building a Partnership with Fourth World Families: The Severely Poor as Leaders”. In Arieh B \& Zionit y eds. Children in Israel on the Threshold of the New Millennium. Jerusalem: National Children's Welfare Council and Ashalim. 